\title{
Double-Tilt in situ TEM holder with multiple electrical contacts and its application in MEMS-based mechanical testing of nanomaterials
}

\author{
Rodrigo A. Bernal ${ }^{1}$, Rajaprakash Ramachandramoorthy ${ }^{1}$, Horacio D. Espinosa ${ }^{1,2}$
}

1. Department of Mechanical Engineering, Northwestern University, Evanston, IL, 60208, USA.

2. iNfinitesimal LLC, Skokie, IL, 60077, USA

\begin{abstract}
MEMS and other lab-on-a-chip systems are emerging as attractive alternatives to carry out experiments in situ the electron microscope. However, several electrical connections are usually required for operating these setups. Such connectivity is challenging inside the limited space of the TEM sideentry holder. Here, we design, implement and demonstrate a double-tilt TEM holder with capabilities for up to 9 electrical connections, operating in a high-resolution TEM. We describe the operating principle of the tilting and connection mechanisms and the physical implementation of the holder. To demonstrate the holder capabilities, we calibrate the tilting action, which has limits of $\pm 15^{\circ}$, and establish the insulation resistance of the electronics to be $36 \mathrm{G} \Omega$, appropriate for measurements of currents down to the nano-amp (nA) regime. Furthermore, we demonstrate tensile testing of silver nanowires using a previously developed MEMS device for mechanical testing, using the implemented holder as the platform for electronic operation and sensing. The implemented holder can potentially have broad application to other areas where MEMS or electrically-actuated setups are used to carry out in situ TEM experiments.
\end{abstract}

\section{Introduction}

In situ Transmission Electron Microscopy (TEM) is recognized as a very powerful technique to establish structure-property relations in materials, as it allows atomic-scale observation of the process of interest as it happens [1]. In fact, this technique has been used to obtain important insights in a variety of physical processes as a function of temperature [2], mechanical stresses [3], electrical stimuli [2], among others.

In particular, several recent breakthroughs have been enabled by in situ TEM in the field of nanomechanics, where the interaction between mechanical variables and the properties of sub-micron specimens is studied (for a recent review see [4]). Some noteworthy examples are the experimental measurement of the strength of carbon nanotubes, in agreement with first-principles calculations [5]; 
experimental confirmation of nanoscale wear as an atom-by-atom stress-assisted chemical reaction [6]; and the "smaller is stronger" set of phenomena, in which yield stress of metallic specimens increases as characteristic size is decreased (for a review see [7]).

All these in situ TEM experiments are enabled by technical breakthroughs in the miniaturization of sensors, actuators and manipulators, which allow the development of testing systems with the ability to fit in the confined space of the TEM chamber and the side-entry specimen holder. Straining holders, piezodriven manipulators and nanoindenters have all been demonstrated [4]. Among these technical approaches, the employment of microelectromechanical systems (MEMS) in situ TEM is emerging as an advantageous alternative as it leverages microfabrication technologies to achieve very precisely dimensioned testing setups, thus allowing carefully-controlled boundary conditions, with the added advantage of electronic actuation and sensing [8]. Furthermore, microfabricated setups not only allow mechanical testing of nanostructures e.g [9], but also multi-physics experiments, which couple thermal [10] or electrical stimuli [11] with structural changes and/or mechanical response.

Typically, several electrical connections to the MEMS are required to operate the actuators and sensors. Making these connections outside the TEM is straightforward using wirebonding technology. However, if the MEMS is to be operated in situ TEM, the space available in the specimen holder imposes challenging constraints [4]. Previously, several single-tilt specimen holders that allow multiple electrical connections have been demonstrated. A system that enables 8 connections has been employed by Petrov and coworkers [12] for operating MEMS that perform rapid heating of nanoparticles and by Espinosa and coworkers for mechanical experiments on nanowires and nanotubes [8]. Other systems allow 4 or 5 connections [13-15].

However, integration of several electrical connections into a double-tilt specimen holder remains challenging and only a few commercial alternatives exist to our knowledge. In particular, companies such as Protochips, Gatan and Nanofactory have developed holders allowing up to 4 electrical connections. All these holders, however, are customized for specific types of chips and provide a low number of connections, thus limiting broader application. For instance, the MEMS device developed by our group for mechanical testing requires at least 5 connections, not counting a ground terminal [16].

In this work, we demonstrate the design and implementation of a double-tilt holder that allows 9 independent electrical connections to a silicon chip located in the tip of the holder. The holder and electronic connections have been designed to have a low profile and therefore allow operation in a JEOL 2100F TEM with a high-resolution pole piece ( $2 \mathrm{~mm}$ gap). Potential for up to 15 connections exists on microscopes with a larger pole-gap. We expect the design to be useful for other applications of MEMS and electronics chips where several electrical connections are needed. To demonstrate the holder capabilities, we use a MEMS system to perform mechanical testing of silver nanowires in situ TEM. 


\section{Materials and Methods}

\section{Holder Design}

The design was conceived for operation in the JEOL 2100F TEM. Fabrication was performed by iNfintesimal LLC, Skokie, LLC. Ferrous or ferromagnetic materials were not employed to fabricate parts located at the holder tip and all materials are vacuum compatible. The main components of the holder tip are a frame, and a tilt-table supported on this frame rotating around the eucentric axis (Figure 1a). The tilt table has an incline in contact with a moving bar (called "push bar" hereon). As the bar moves back and forth against the incline, it causes rotation of the tilt table (Figure 1b). A torsion spring pushes the tilt table against the push bar, so that contact is maintained (Figure 1a). The geometry of the incline and the radius of the bar's tip determine the relation between linear displacement of the bar and rotation of the table, as well as the maximum tilt angle. The specific design parameters are shown in the Results section. The tilt table has a u-shape, a rectangular slot and a set screw, in order to accommodate the electronic assembly that is used to operate the MEMS (Figure 1c).

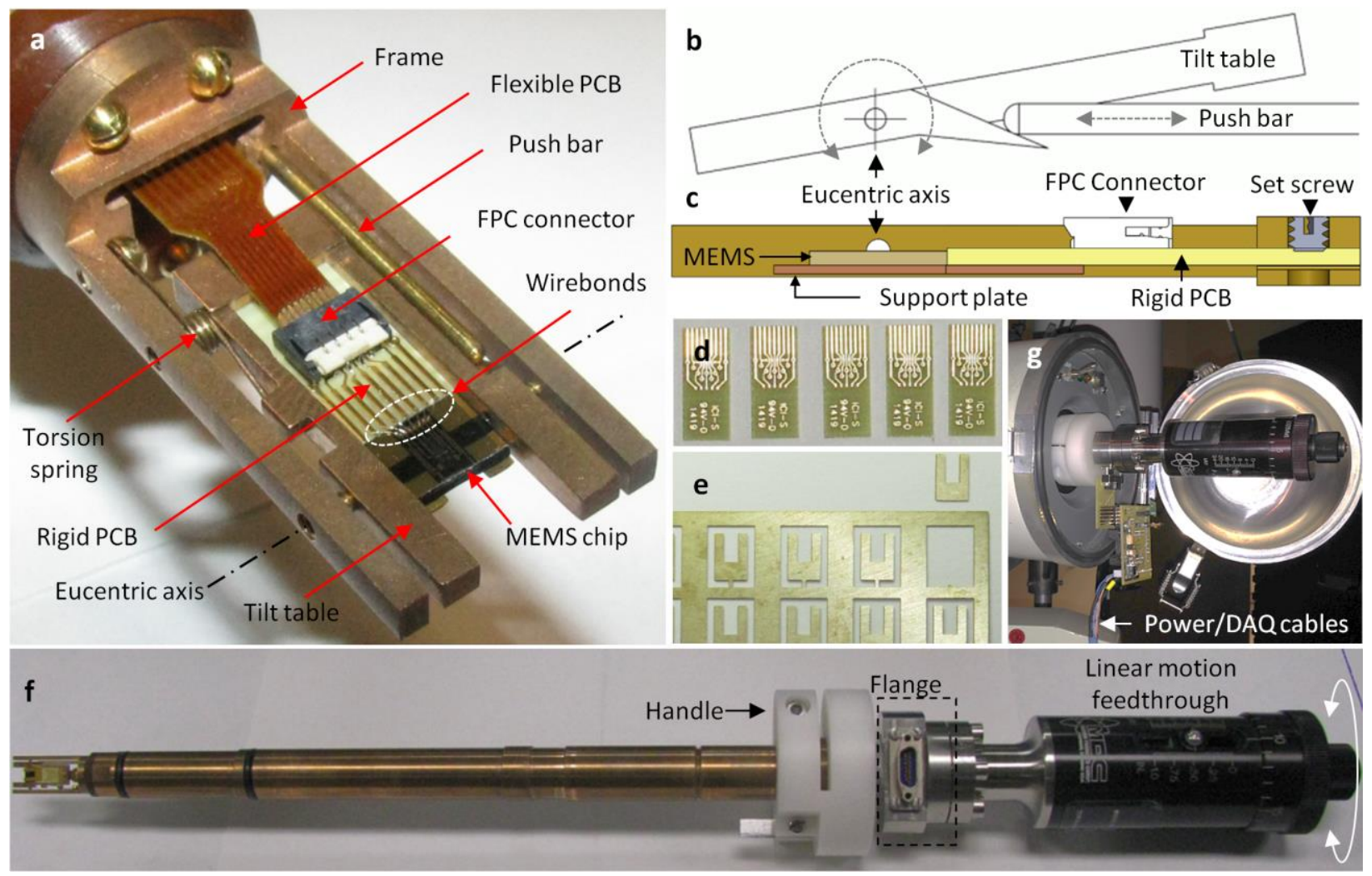

Figure 1: Schematics and pictures of the double-tilt holder. a) Picture of the holder tip. b) Schematic of the tilting mechanism. The push bar, moving back and forth, creates rotation in the tilt table. c) Cross-section of the electronic assembly and how it fits in the tilt table. d) Pictures of the rigid boards and e) support plates f) Picture of the complete holder. g) Picture of the holder inserted in the JEOL 2100F TEM and connected to the MEMS electronics. 
The electronic assembly is positioned on the tilt table, and allows the connection from the outside electronics to the MEMS chip. A custom-fabricated rigid printed circuit board (PCB) has several parallel traces finished with gold to allow wirebonding to the MEMS chip. The MEMS has a width of 5mm and a thickness of $0.41 \mathrm{~mm}$; the latter dimension is typical of microfabricated devices. The PCB is also thin $(0.46 \mathrm{~mm})$ and was fabricated with low-outgassing FR4 epoxy composite (Figure 1a,d). The chip and board are joined with the aid of a thin $(0.25 \mathrm{~mm})$ brass support plate (Figure 1c,e), electrochemically etched from a plate. The chip, PCB and support are bonded together with high-vacuum-compatible epoxy (Epo-tek 353ND). Both board and support plate can be manufactured in large quantities with reasonable cost and are therefore consumables (Figure 1d-e). One advantage of the design is that only the MEMS chip goes in between the pole pieces during insertion and operation of the holder. Furthermore, the assembly was designed so that the specimen position is centered nominally on the tilting axis (note that in Figure 1a and c, the MEMS is bisected by the eucentric axis). Note that other type of electronic chips could be accommodated by redesigning only the electronic assembly, not the whole holder; therefore, allowing potential broad usage of the setup for other applications.

The rigid board, aside from having traces for wirebonding, has terminals to allow the reflow-soldering of a commercially-available, low-profile, high density Flex-printed-circuit (FPC) connector (Panasonic AYF330935). The connector is made of vacuum-compatible UL94V-0 resin. This connector has a pitch of $0.6 \mathrm{~mm}$ and therefore allows 9 connections without being wider than $5 \mathrm{~mm}$, which is the width between the prongs of the tilt table. The height above the rigid board is of $0.9 \mathrm{~mm}$, essential to maintain the low profile of the setup. These FPC connectors are typically used in the manufacture of smartphones and tablets, and therefore a variety of geometries and connection densities are available. In particular, if greater height is allowed with respect to the board, up to 15 connectors could be accommodated. Other MEMS chip designs of smaller size could even free up more space and allow for more connections.

The FPC connector interfaces with a custom-designed flexible PCB (Figure 1a), composed of copper conductors patterned on top of vacuum-compatible kapton polymer. A high mechanical compliance of this board is important so that it does not hinder rotation of the tilt-table. The flexible PCB extends into the hollow holder shaft, and interfaces at the other end of the holder (Figure 1f) with a commercial, hermetic, 15-pin connector (Glenair, Glendale, CA) located in a custom-machined stainless steel flange. The flange is bonded to the holder shaft using Torr-seal cement.

On the other hand, the tilting mechanism, as alluded to before, is actuated by a push-bar. The push bar is coupled to an internal rod, located inside the hollow holder shaft. This rod is in turn coupled to a linearmotion, ultra-high-vacuum feedthrough, which bolts to the flange and provides precise linear motion $(0.025 \mathrm{~mm}$ resolution) by rotating a knob (Figure 1f). This feedthrough is available commercially (MDC Vacuum 660000, Hayward, CA). 


\section{Results and Analysis}

\section{Tilt-angle calibration}

The angle of the incline in the tilt-table and the radius of the push-bar determine the angular motion of the tilt-table as a function of linear motion of the push bar. The dimensions of this incline were determined by carrying out kinematic simulations using commercial software (Working Model). In order to calibrate the holder before operation in the TEM, the holder tip was placed under a camera equipped with a macro lens, and the tilt angles were measured from pictures taken at several positions of the push bar, moved using the linear feedthrough. The linear motion of the push bar is known by markings in the feedthrough knob (Figure 2e inset), where 1 division equals $0.025 \mathrm{~mm}$ of motion. Through this optical calibration, a given position of the knob can be associated with a particular tilt angle, providing knowledge of said quantity while in TEM operation. The experimental results are presented in Figure 2. The experimental and designed motion curves match well, which indicates good precision on the machined components. Note that the current design allows tilts up to -14.7 to +15 degrees. However, using the same design concept and a different tilt table with a steeper incline, higher tilts could be achieved, for instance, if one desires operation in a microscope where the pole-gap is larger. 

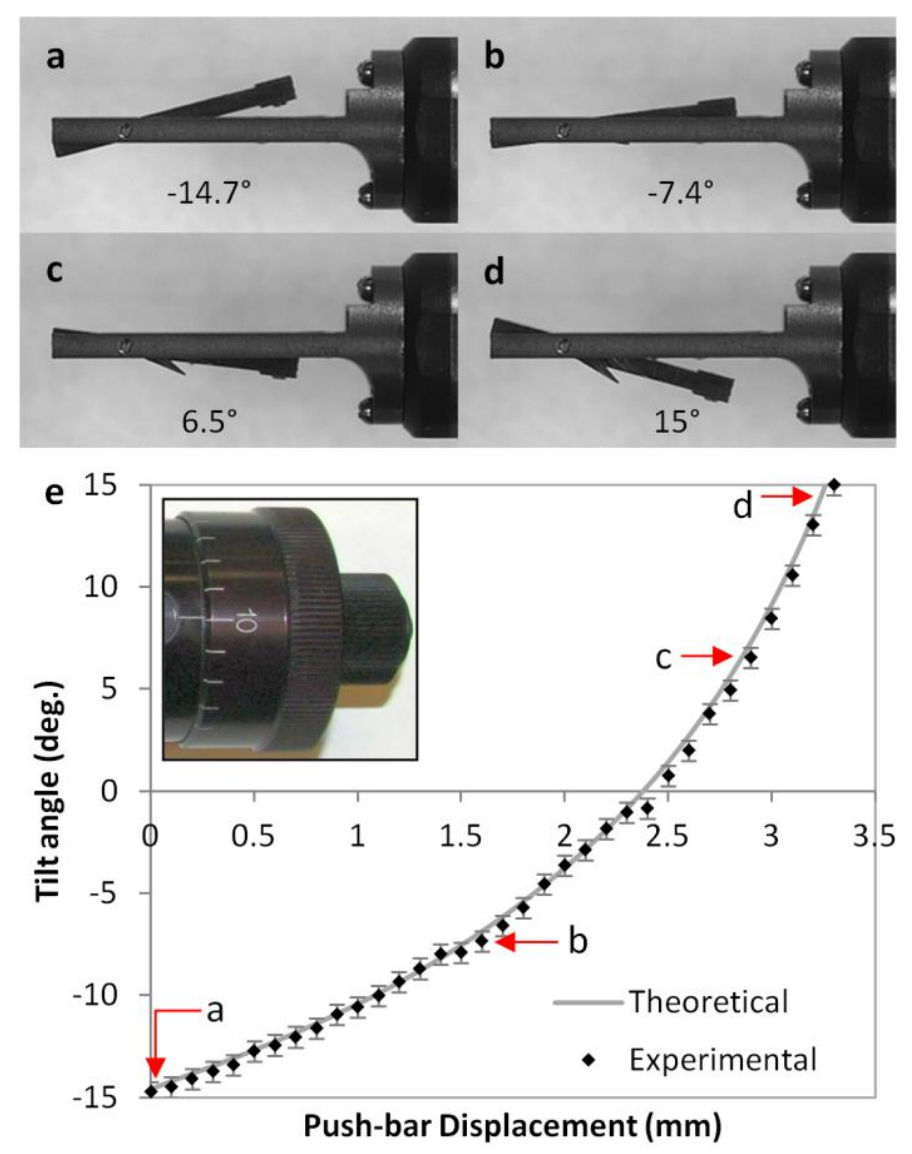

Figure 2: Tilt angle calibration. a-d: Pictures of several instances of tilt. e) Calibration curve. The data points corresponding to a$\mathrm{d}$ are indicated. Inset shows the knob in the feedthrough used for this calibration, where 1 division corresponds to $0.025 \mathrm{~mm}$ linear motion.

The relation between linear motion and tilt angle is nonlinear. The data in Figure $2 \mathrm{e}$ were taken every 4 divisions of the graded scale in the rotating knob of the linear feedthrough. Although the mechanism is not discrete, if we take one division of the linear motion as representative of the resolution of the mechanism, the resulting tilt angle resolution is $0.06^{\circ}$ near $-15^{\circ}$, and becomes progressively coarser as tilt angle increases, reaching a value of $\sim 0.5^{\circ}$ near $+15^{\circ}$.

\section{Electrical Performance: Insulation Resistance}

Due to the closeness of the traces in the flexible PCB, it is important to assess the insulation resistance between them. This measurement provides bounds for the minimum resolvable currents in our setup, important for its applicability in other areas, for example to measure currents in nanostructures [11], which are of the order of nano Amperes (nA). To measure this resistance, a Keithley 4200 semiconductor characterization system was used to apply voltages between two adjacent traces, and to measure the current passing between them. The results are shown in Figure 3. The insulation resistance is found to be $36 \mathrm{G} \Omega$, with a maximum current of about $130 \mathrm{pA}$ passing at $+5 \mathrm{~V}$. This insulation is very good for most 
electrical measurements, and implies a practical resolution for current measurements of about 1.3nA (10 times the insulation resistance).

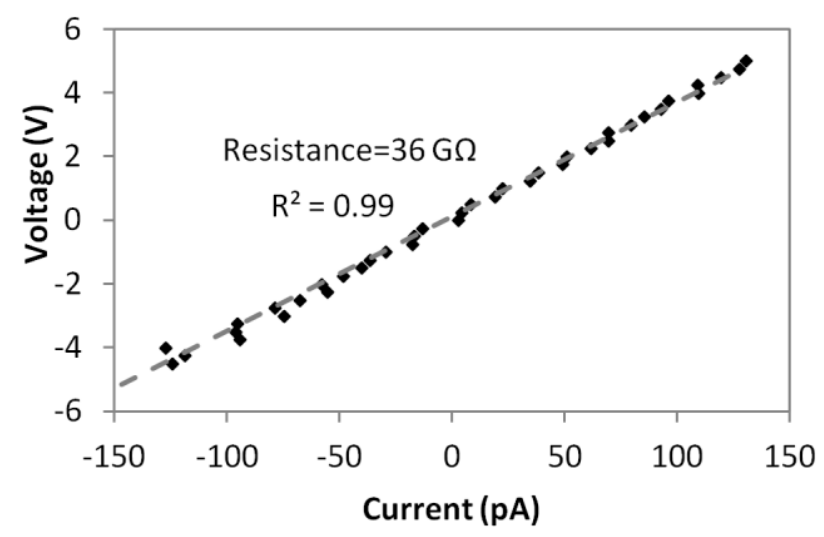

Figure 3: Insulation resistance of adjacent traces in the setup.

\section{In situ TEM experiments with MEMS for Mechanical Testing of Nanostructures}

In previous work, our group has developed a MEMS device for mechanical tensile testing of nanostructures $[17,18]$. In this system, a thermal actuator (Figure 4a) pulls on one end of the specimen (Figure 4b). At the other end, the specimen is attached to a load sensor (Figure 4a), which measures the force in the specimen. For calibration purposes, devices with a rigid joint between actuator and sensor are also fabricated (Figure 4c). Note that the device also has two separate connections to the specimen ("specimen traces"), which can be used to measure electrical properties of the specimens as a function of applied stress. 

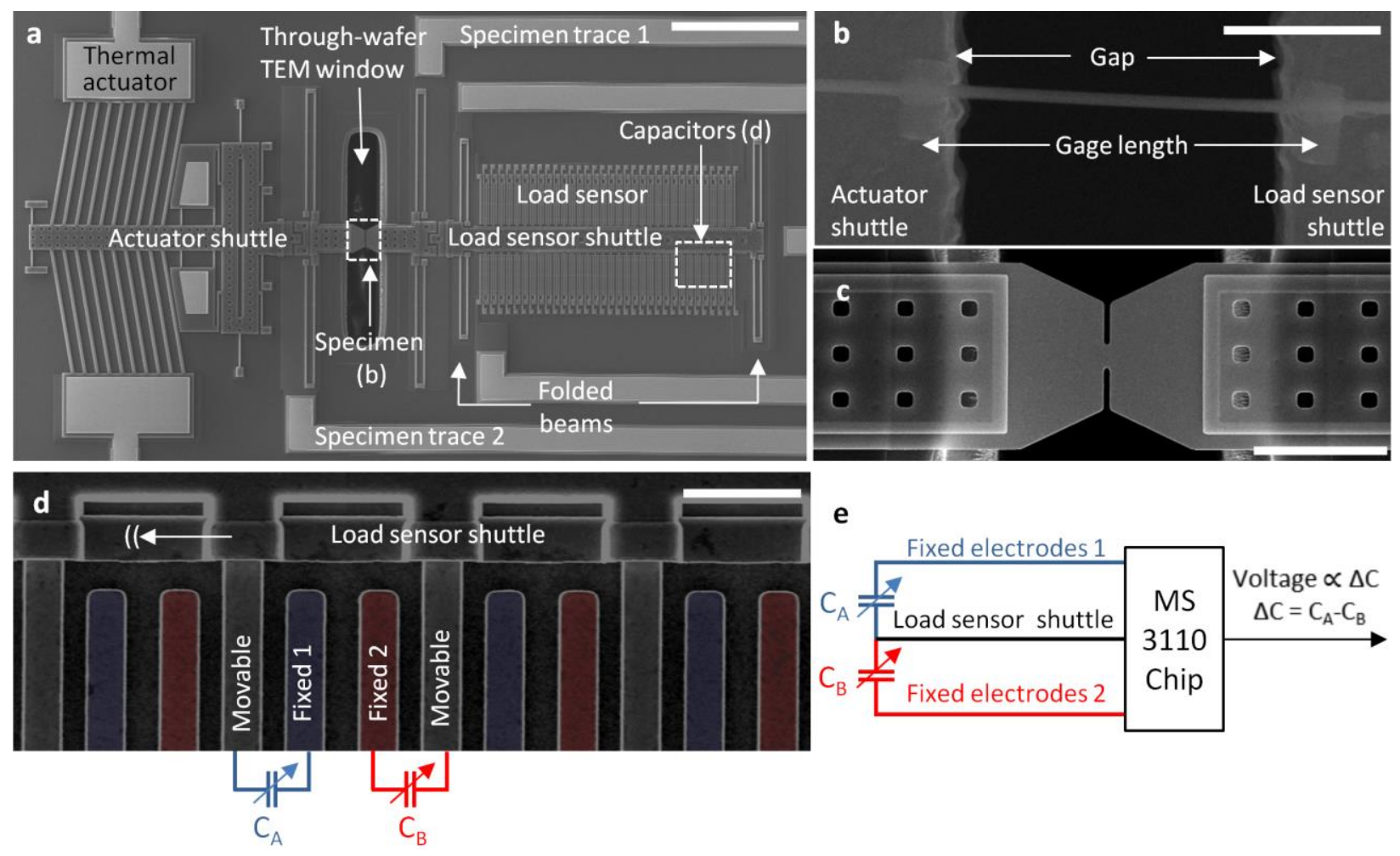

Figure 4: MEMS for mechanical testing of nanowires. a) SEM image of the device, scale bar $150 \mu \mathrm{m}$ b) Specimen mounted on the device gap, scale bar $1 \mu \mathrm{m}$ c) Device with a rigid joint between sensor and actuator, used for calibrating motion of sensor and actuator Vs. voltages (See text and Figure 5) (scale bar: $50 \mu \mathrm{m}$ ). d) Detail of the capacitive sensor embedded in the load sensor, scale bar $10 \mu \mathrm{m}$. e) Electrical schematic of the capacitive sensing scheme used to measure load sensor displacement.

In order to obtain mechanical measurements with these MEMS, i.e. an engineering stress-strain curve, there are several requirements. A detailed explanation is given elsewhere [16, 17]; here we provide a short overview. For engineering stress, measurement of the force in the specimen and its cross section are needed. For engineering strain, specimen deformation and its initial gage length need to be measured.

The force in the specimen can be obtained by multiplying the displacement of the load sensor (measured during the test) by its stiffness, which is controlled by the dimensions of the supporting folded beams. This stiffness is known from calibration. The displacement is obtained electronically through the capacitive sensor embedded in the structure (Figure 4d), which consists of three sets of electrodes, all together composing a set of differential capacitors i.e. two variable capacitors with a common electrode. One set of electrodes, the common, moves alongside the load sensor shuttle, while the two other sets are stationary. Upon movement, the capacitance between the common electrode and the fixed electrodes does change. This differential capacitance change can be converted to a voltage by a commercial chip (MS3110) (Figure 4e) [17]. The voltage output is proportional to the capacitance change, which is in turn proportional to the load sensor displacement. Finally, stress on the specimen can be obtained by dividing the force by the specimen cross-section, which is measured in TEM. 
For measurement of strain, specimen deformation and initial specimen length can be obtained by TEM and SEM imaging respectively. Specimen deformation can be assumed to be equal to the change in gap between the shuttles (Figure 4b) [19] and can be determined by digital image correlation of TEM images, where the position of the shuttles is tracked across images or videos taken at subsequent straining steps. Another way of obtaining the specimen deformation is electronically, by knowing the displacement of the actuator (determined from its applied voltage and calibration, see below) and subtracting the displacement of the load sensor (known from the electronics). The strain can finally be determined by dividing the specimen deformation by the gage length (Figure 4b).

The thermal actuator (Figure 4a) consists of several chevron silicon beams connected to a central shuttle. When a voltage is applied, current flow creates Joule heating in the silicon, triggering thermal expansion of the chevron beams, and therefore driving the shuttle to the left (in Figure 4a). Calibration of the actuator can be done by applying known voltages and measuring the displacement with the load sensor, in the device that has a solid connection (Figure 4c).

In order to verify that our TEM holder establishes appropriate electrical connections to the MEMS, we connected the device with a rigid joint between actuator and sensor (Figure $4 \mathrm{c}$ ) to the holder. Note that this device requires 7 connections plus a ground connection. Specifically, 2 connections are needed for the thermal actuator, 3 for the load sensor, and 2 for the specimen terminals (in this case grounded). Unused terminals are connected to circuit ground to prevent charge accumulation. To ensure lower-noise behavior, circuit ground (a trace available in the rigid PCB) is also connected to the holder body.

While the device is inside the TEM, we apply a triangular wave to the actuator and record the output of the load sensor. From previous work, it is known that the actuator has a nearly-quadratic behavior as a function of applied voltage [20]. This can be seen in the response curve of the load sensor, which is a periodic nonlinear curve, as shown in Figure 5a. Plotting the voltage output of the load sensor (proportional to displacement of the rigidly-coupled actuator and sensor system) as a function of the actuator input voltage for half a cycle shows the nearly quadratic response of the actuator (Figure 5b). 

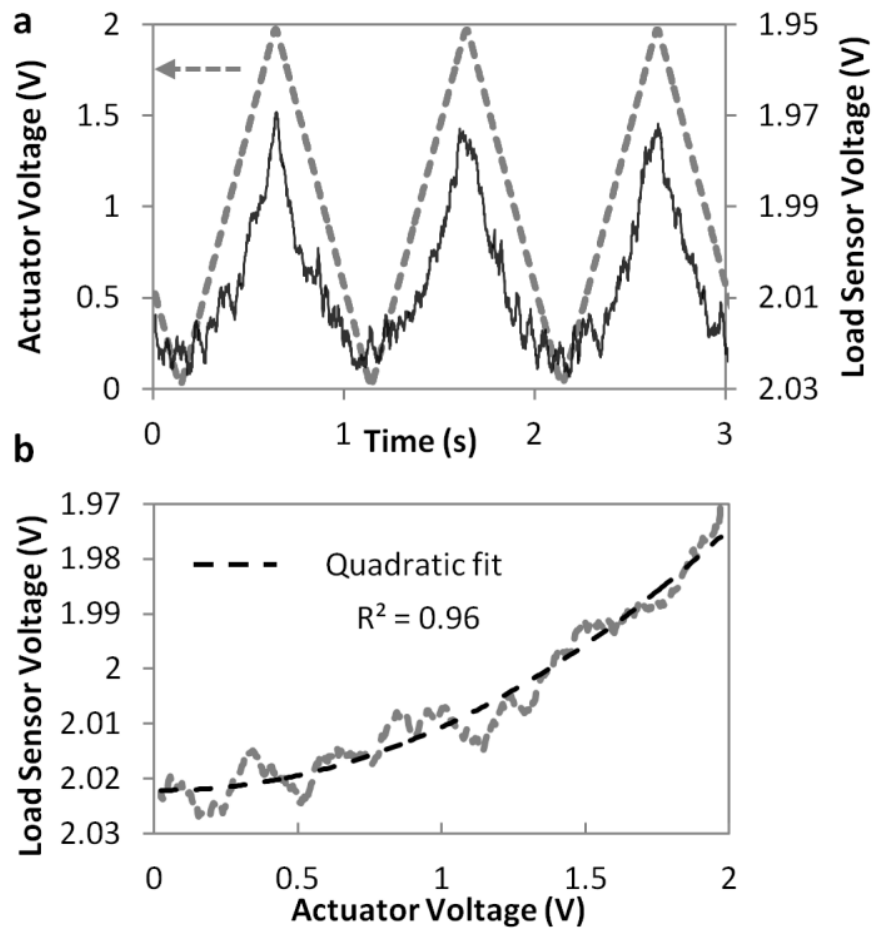

Figure 5: Electronic testing of the holder with MEMS for mechanical testing. See text for further explanation a) Actuator voltage and load sensor voltage as a function of time. A linear input generates a quadratic output, as expected. b) Plot of half a cycle showing the quadratic response of the load sensor voltage.

Having established the electronics behave appropriately, we carried out experiments with the device for nanowire testing. This device has a gap between load sensor and actuator to position the specimen (Figure 4b). Details about specimen preparation are given elsewhere [4]. In particular, we verified the tilting action is occurring by actuating the vacuum feedthrough and looking at the device in the TEM at low magnification. As can be seen in Figure $6 a$ and $b$, the shuttles appear to move with respect to the TEM window, which is a result of the change in their projected image as the device is tilted with respect to the TEM beam.

Furthermore, we carried out a tensile test for a $73 \mathrm{~nm}$-diameter penta-twinned silver nanowire. These nanowires are composed of five single-crystal domains, oriented on the $<110\rangle$ directions, and intersecting at $\{111\}$ twin planes (for more details about the crystal structure see [21]). As such, the obtained diffraction pattern (Figure 6d) is a superposition of the $\{111\}$ and $\{110\}$ zone axes of the FCC structure for silver. This diffraction pattern was captured by tilting $0.5^{\circ}$ with the primary tilt (goniometer tilting) and 1 division with the holder knob for secondary tilt $\left(\sim 0.5^{\circ}\right)$. Note the improvement in alignment, compared to the diffraction pattern obtained without tilting (Figure 6c), demonstrating the advantage of carrying out experiments with the double-tilt holder.

Subsequently, we carry out a tensile test of the nanowire up to fracture (Figure 6e-f) by applying a monotonically increasing voltage waveform to the thermal actuator and capturing the voltage in the load 
sensor. These data allows us to calculate the stress-strain curve as explained above. The resulting stressstrain curve for the nanowire is shown in Figure $6 \mathrm{~g}$. The modulus of the specimen is $91 \mathrm{GPa}$ (computed with the data up to $1 \%$ strain), which agrees well with our previous results in this material [21] that indicate a size effect i.e. an increase of the modulus with respect to the bulk value (84 GPa) [22].
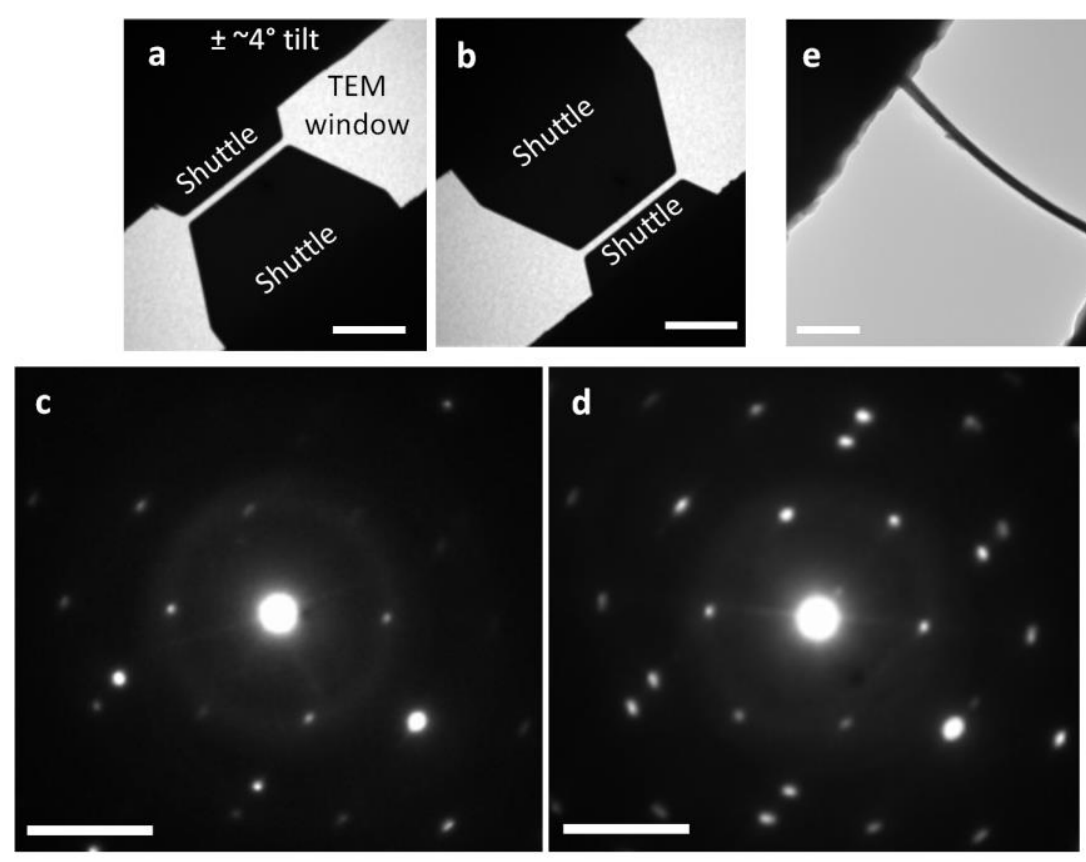
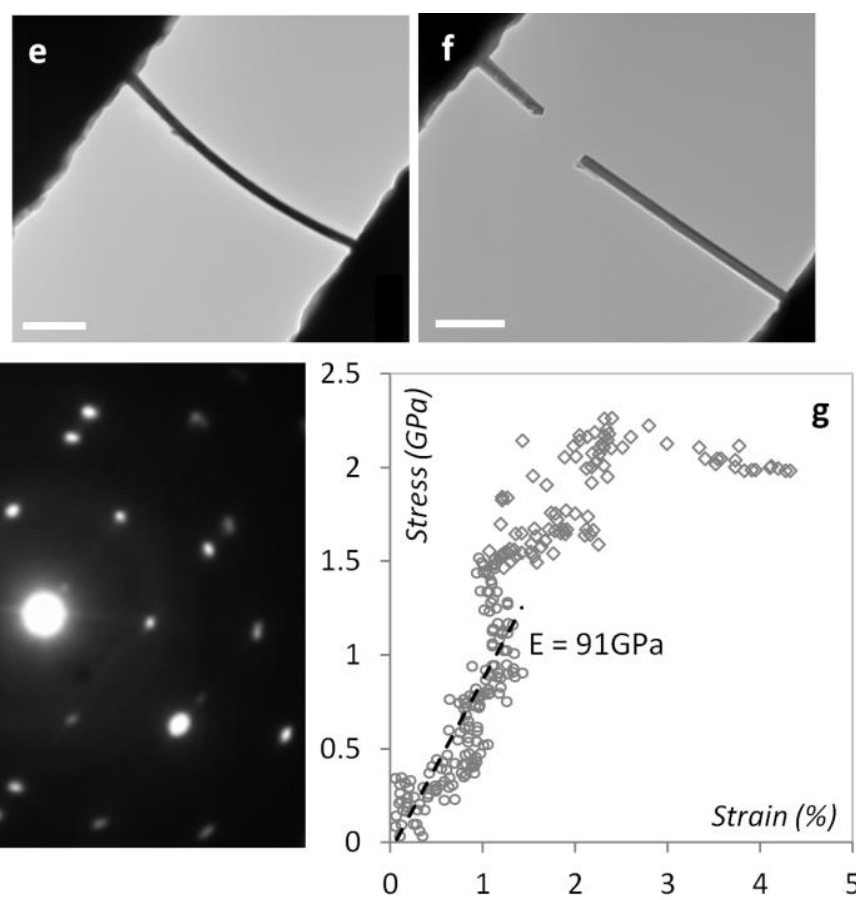

Figure 6: a,b) Tilting of the MEMS device. As the device is tilted by the holder respect to the electron beam, changes in the projected image of the shuttles can be observed. Scale bars $10 \mu \mathrm{m}$. c) Diffraction pattern of the tested penta-twinned silver nanowire before tilting. d) Diffraction pattern after optimizing primary and secondary tilt. Scale bars: $51 / \mathrm{nm}$. e) Image of the nanowire before testing. f) Image of the nanowire just after fracture. Scale bars: $500 \mathrm{~nm}$. g) Stress-strain curve for the tested nanowire.

\section{Conclusions}

In summary, we have designed and implemented a TEM specimen holder with capabilities of $15^{\circ}$ double-tilt and up to 9 electrical connections, able to operate in the JEOL 2100F TEM equipped with the high resolution pole piece ( $2 \mathrm{~mm}$ gap). Potential for extending the connectivity up to 15 connections exists if the holder is operated in a TEM with larger pole piece gap. We demonstrated appropriate electrical insulation between adjacent traces, which enables precision electrical measurements, and correct connectivity by actuating and sensing electronic signals for a MEMS device used for mechanical testing of nanostructures. Tilting capabilities and a tensile test of a $73 \mathrm{~nm}$-diameter silver nanowire were demonstrated as well.

We expect the implementation of this holder to be beneficial for other fields where MEMS or silicon chips are being used for other type of in situ TEM studies that require several electrical connections, such 
as heaters for thermal studies, or microfabricated stages for electrical probing of specimens. The design is quickly adaptable to other type of silicon chips by redesigning the rigid PCB. Since wirebonding is employed to contact the silicon chip, successful operation of a variety of electronic chips should be straightforward.

\section{Acknowledgements}

H.D.E acknowledges support of the Army Research Office through DURIP instrumentation award No. W911NF-12-1-0366 and NSF through award No. DMR-0907196. We thank Dr. Juan P. Giraldo from iNfinitesimal LLC for mechanical assembly and Prof. Wilbur Bigelow at University of Michigan for very useful discussions on the holder design.

\section{References}

1. Ferreira, P.J., K. Mitsuishi, and E.A. Stach, In situ transmission electron microscopy. Mrs Bulletin, 2008. 33(2): p. 83-85.

2. Howe, J.M., H. Mori, and Z.L. Wang, In situ high-resolution transmission electron microscopy in the study of nanomaterials and properties. Mrs Bulletin, 2008. 33(2): p. 115-121.

3. Legros, M., D.S. Gianola, and C. Motz, Quantitative In Situ Mechanical Testing in Electron Microscopes. Mrs Bulletin, 2010. 35(5): p. 354-360.

4. Espinosa, H.D., R.A. Bernal, and T. Filleter, In Situ TEM Electromechanical Testing of Nanowires and Nanotubes. Small, 2012. 8(21): p. 3233-3252.

5. Peng, B., et al., Measurements of near-ultimate strength for multiwalled carbon nanotubes and irradiation-induced crosslinking improvements. Nature Nanotechnology, 2008. 3(10): p. 626-631.

6. Jacobs, T.D.B. and R.W. Carpick, Nanoscale wear as a stress-assisted chemical reaction. Nature Nanotechnology, 2013. 8(2): p. 108-112.

7. Greer, J.R. and J.T.M. De Hosson, Plasticity in small-sized metallic systems: Intrinsic versus extrinsic size effect. Progress in Materials Science, 2011. 56(6): p. 654-724.

8. Haque, M.A., H.D. Espinosa, and H.J. Lee, MEMS for In Situ Testing-Handling, Actuation, Loading, and Displacement Measurements. Mrs Bulletin, 2010. 35(5): p. 375-381.

9. Guo, H., et al., Mechanics and Dynamics of the Strain-Induced M1-M2 Structural Phase Transition in Individual VO2 Nanowires. Nano Letters, 2011. 11(8): p. 3207-3213.

10. Vincent, L., et al., Novel Heterostructured Ge Nanowires Based on Polytype Transformation. Nano Letters, 2014. 14(8): p. 4828-4836.

11. Bernal, R.A., et al., In Situ Electron Microscopy Four-Point Electromechanical Characterization of Freestanding Metallic and Semiconducting Nanowires. Small, 2014. 10(4): p. 725-733.

12. Zhang, M., et al., In situ transmission electron microscopy studies enabled by microelectromechanical system technology. Journal of Materials Research, 2005. 20(7): p. 18021807.

13. $\mathrm{Xu}, \mathrm{S} . \mathrm{Y} ., \mathrm{J} . \mathrm{Xu}$, and M.L. Tian, A low cost platform for linking transport properties to the structure of nanomaterials. Nanotechnology, 2006. 17(5): p. 1470-1475.

14. $\mathrm{Xu}, \mathrm{T} . \mathrm{T}$., et al., A platform for in situ multi-probe electronic measurements and modification of nanodevices inside a transmission electron microscope. Nanotechnology, 2014. 25(22).

15. Borrnert, F., et al., A cheap and quickly adaptable in situ electrical contacting TEM sample holder design. Ultramicroscopy, 2014. 139: p. 1-4. 
16. Espinosa, H.D., Y. Zhu, and N. Moldovan, Design and operation of a MEMS-based material testing system for nanomechanical characterization. Journal of Microelectromechanical Systems, 2007. 16(5): p. 1219-1231.

17. Y. Zhu, and H.D. Espinosa, An Electromechanical Material Testing System for In situ Electron Microscopy and Applications. Proceedings of the National Academy of Sciences of the United States of America, 2005. 102(41): p. 14503-14508.

18. Y. Zhu, N. Moldovan, and H.D. Espinosa, A Microelectromechanical Load Sensor for In Situ Electron and X-ray Microscopy Tensile Testing of Nanostructures. Applied Physics Letters, 2005. 86(1): p. 013506.

19. Agrawal, R., et al., Elasticity Size Effects in ZnO Nanowires: A Combined ExperimentalComputational Approach. Nano Letters, 2008. 8(11): p. 3668-3674.

20. Zhu, Y., A. Corigliano, and H.D. Espinosa, A thermal actuator for nanoscale in situ microscopy testing: design and characterization. Journal of Micromechanics and Microengineering, 2006. 16(2): p. 242-253.

21. Filleter, T., et al., Nucleation-Controlled Distributed Plasticity in Penta-twinned Silver Nanowires. Small, 2012. 8(19): p. 2986-2993.

22. Zhu, Y., et al., Size effects on elasticity, yielding, and fracture of silver nanowires: In situ experiments. Physical Review B, 2012. 85(4). 